\title{
Breast microcalcifications: Past, present and future (Review)
}

\author{
ANGELA F. LOGULLO ${ }^{1,2}$, KARLA C.K. PRIGENZI ${ }^{2}$, CRISTIANE C.B.A.NIMIR ${ }^{2}$, \\ ANDREIA F.V. FRANCO ${ }^{1}$ and MARIO S.D.A. CAMPOS ${ }^{3}$ \\ ${ }^{1}$ Department of Pathology, Paulista School of Medicine, Federal University of São Paulo (UNIFESP), São Paulo 04023-062; \\ ${ }^{2}$ Department of Pathology and ${ }^{3}$ Breast Imaging Service, Femme Laboratories, São Paulo 04004-030, Brazil
}

Received June 22, 2021; Accepted October 19, 2021

DOI: $10.3892 / \mathrm{mco} .2022 .2514$

\begin{abstract}
Mammary microcalcifications (MCs) are calcium deposits that are considered as robust markers of breast cancer when identified on mammography. MCs are frequently associated with premalignant and malignant lesions. The aim of the present review was to describe the MC types and associated radiological and pathological aspects in detail, provide insights and approaches to the topic, and describe specific clinical scenarios. The primary MC types are composed of calcium oxalate, hydroxyapatite and hydroxyapatite associated with magnesium. The first type is usually associated with benign conditions, while the others remain primarily associated with malignancy. Radiologically, MCs are classified as benign or suspicious. MCs may represent an active pathological mineralization process rather than a passive process, such as degeneration or necrosis. Practical management of breast specimens requires finely calibrated radiological pathological procedures. Understanding the molecular and structural development of MCs may contribute to breast lesion detection and treatment.
\end{abstract}

\section{Contents}

1. Introduction

2. Microscopic localization of MCs

3. Etiology of MCs

4. Radiological classification of MCs

5. MC management and biopsy with radiological guidance

6. Sending MC specimens to the pathology laboratory

7. MCs in breast cancer

8. MCs in specific clinical scenarios

9. Conclusions

Correspondence to: Professor Andreia F.V. Franco, Department of Pathology, Paulista School of Medicine, Federal University of São Paulo (UNIFESP), 740 Botucatu Street, São Paulo 04023-062, Brazil

E-mail: afvfranco@gmail.com

Key words: breast cancer, mammogram, microcalcifications, breast diagnosis, radiological and pathological aspects

\section{Introduction}

Mammary microcalcifications (MCs) are deposits $<0.5 \mathrm{~mm}$ in diameter within the breast tissue. This radiological feature was first described by Albert Salomon, a German surgeon who imaged $>3,000$ surgical specimens in an attempt to describe the association of MCs with breast cancer and tumor spread to lymph nodes (1). In diagnostic mammograms, MCs were first described in 1951 and became progressively crucial in cancer detection. Currently, 30-50\% of non-palpable breast cancers are detected solely by MCs revealed by mammography (2-4).

The presence of MCs in mammograms strongly suggests premalignant or malignant lesions. The type and composition of MCs are crucial for risk stratification. For example, pleomorphic or fine linear MCs are strongly associated with malignancy, as demonstrated in a meta-analysis involving $>10,000$ patients (5).

Most breast calcifications are dystrophic and appear in the terminal ductal-lobular units, and they associated with various pathological processes, such as fluid collections in cysts, inflammation, infection, and benign or malignant tumors $(6,7)$. The aim of the present review was to provide details regarding the types of MCs and associated radiological and pathological aspects, in order to provide insights and practical approaches to the topic, including specific clinical scenarios.

\section{Microscopic localization of MCs}

Histologically, MCs are calcium-related deposits in the mammary ductal-lobular units associated with an epithelial component, such as microcystic adenosis, sclerosing adenosis, cystic lesions and proliferative lesions (6). MCs may also be entrapped within a mesenchymal component, either in the stroma (stromal reaction within a malignant tumor) or they may appear as fibrous lesions with a connective component (fibroadenoma), a fibrotic healing process, or steatonecrosis (Fig. 1) (6).

At the biochemical level, MCs are generally classified into two main types: Type I, composed of calcium oxalate (CO), and type II, composed of hydroxyapatite (HA). The classification is based on chemical composition and mammographic characteristics, including morphology, distribution and density (Table I) (8-10). The difference between types is significant because evidence suggests that type II is often associated with malignant lesions (11). 
Table I. Description of differences among type I, II and Mg-HA of MCs according to distinct features.

\begin{tabular}{|c|c|c|c|c|}
\hline Features of MCs & Type I & Type II & $\mathrm{Mg}-\mathrm{HA}$ & Refs. \\
\hline Composition & $\mathrm{CO}\left(\mathrm{CaC}_{2} \mathrm{O}_{4} 2 \mathrm{H}_{2} \mathrm{O}\right)$ & Calcium HA $\left[\mathrm{Ca}_{10}\left(\mathrm{PO}_{4}\right)_{6}(\mathrm{OH})_{2}\right]$ & $\mathrm{Mg}-\mathrm{HA}\left[\mathrm{Mg}_{10}\left(\mathrm{PO}_{4}\right)_{6}(\mathrm{OH})_{2}\right]$ & (8) \\
\hline Association with lesions & Benign disease & Benign and malignant disease & Mostly malignant disease & (8) \\
\hline Carbonate & Not present & $\begin{array}{l}\text { More carbonate in the } \\
\text { malignant the lesion }\end{array}$ & Data not available & (9) \\
\hline Color in optical microscopy & Amber or transparent & Opaque, grey/white & Opaque, grey/white & (9) \\
\hline $\begin{array}{l}\text { Birefringence in polarized } \\
\text { light microscopy }\end{array}$ & Birefringent & Non-birefringent & Non-birefringent & (9) \\
\hline $\begin{array}{l}\text { Experimental effect in } \\
\text { breast cancer cell lines }\end{array}$ & $\begin{array}{l}\text { Not capable of } \\
\text { inducing motility }\end{array}$ & Capable of inducing motility & Data not available & (13) \\
\hline
\end{tabular}

MCs, microcalcifications; $\mathrm{CO}$, calcium oxalate; HA, hydroxyapatite; Mg-HA, magnesium-substituted HA.

A
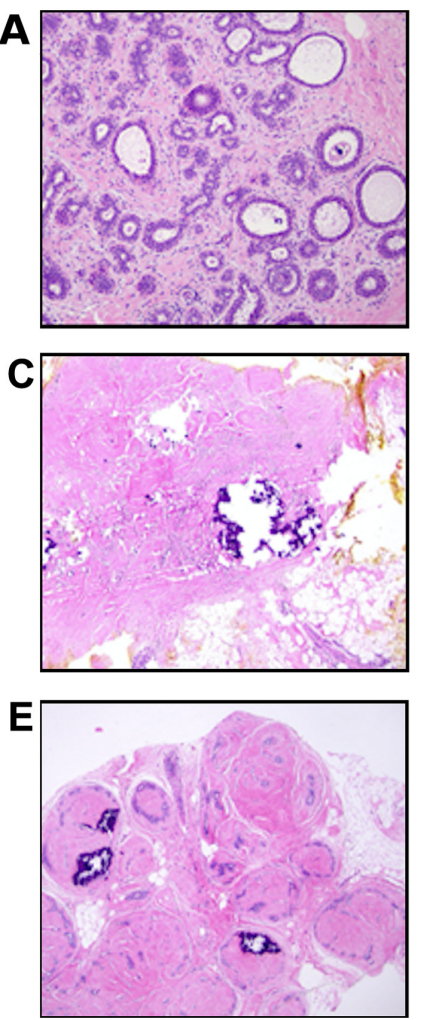
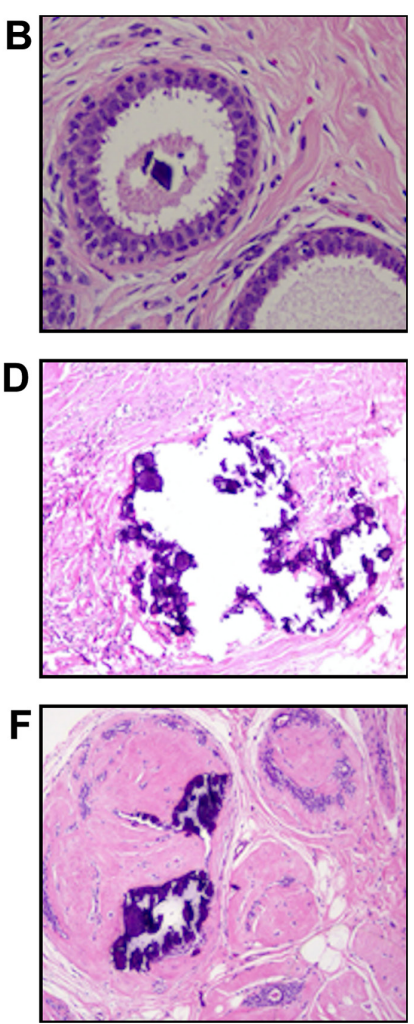

Figure 1. Histopathological representation of MCs within different types of breast lesions. (A and B) MCs associated with columnar cell lesion (HE staining; magnification, $\mathrm{x} 200$ ); (C and D) Steatonecrosis and dystrophic calcifications [HE staining; magnification, (C) x200 and (D) x400]. (E and F) Fibroadenoma showing stromal MCs [HE staining; magnification, (E) x100 and (F) x200]. MCs, microcalcifications.

$\mathrm{CO}$ is produced by apocrine cells in the breast and is frequently associated with benign breast tissue alterations. $\mathrm{CO}$ cannot be metabolized by mammalian cells, and evidence suggests that its presence may metabolically affect epithelial cells, as it was shown to induce proliferation and c-Fos overexpression in MCF-7 cells (12).

Type II MCs may be associated with benign and malignant breast entities; they are present in a wide range of benign

entities, such as fibroadenomas, fibroadenosis and sclerosing adenosis, and may be partially attributed to two mechanisms that are also associated with malignant invasive cancer in experimental models, namely necrosis and fibrosis $(9,13)$. Collagen type I is commonly associated with osteogenesis, and it is a significant component of benign nodular lesions, such as fibroadenomas and areas of fibroadenomatoid changes. Indeed, detection of type II collagen in these lesions is common in clinical practice (10).

Another morphological feature present in benign as well as malignant entities is macrophage recruitment. It has been reported that tumor-associated macrophages (TAMs) that secrete cytokines play a pivotal role in MCs. Cysts, areas exhibiting damage, such as adiponecrosis or inflammation, and previous surgical procedures are associated with MCs and they are usually densely infiltrated with macrophages (14).

Recently, a novel third biochemically distinct form of MC has been described as magnesium-substituted HA (Mg-HA). $\mathrm{Mg}-\mathrm{HA}$ and type II MCs are robustly associated with malignancy (15). Scimeca et al (15) observed that CO MCs were associated with benign lesions in $81.8 \%$ of the cases (18 of 22), whereas $97.7 \%$ (43 of 44) of malignant lesions displayed complex forms of MCs, primarily composed of a different form of HA, namely $\mathrm{Mg}-\mathrm{HA}$; this form was present only in malignant lesions (23 of 44).

\section{Etiology of MCs}

Dystrophic calcification is traditionally considered a passive feature associated with cell degeneration and necrosis and, therefore, is considered to be a passive and unspecific process (16). However, breast MCs are under pathophysiological scrutiny. While type I MCs are usually associated with cysts or fat necrosis (a representative example of passive deposits), type II MCs are more frequently associated with abnormal epithelial cells (16).

Bone HA deposition represents an active cell-mediated process (17) and is part of physiological mineralization. However, breast MCs occur outside the skeleton and, therefore, are considered to represent a pathological mineralization process. Moreover, evidence suggests that MCs may be 
Table II. Distinct features of typical benign and malignant-related MCs in mammograms.

\begin{tabular}{lcc}
\hline Characteristics & Benign breast lesions & Malignant breast lesions \\
\hline MCs & Spaced & Compact clusters \\
Amount of carbonate & Higher & Lower \\
Shape & Ring-shaped & Vermicular, casting-type \\
Protein matrix/mineral ratio & High & Low \\
Rate of Mg substitution & Lower & Higher
\end{tabular}

MCs, microcalcifications.

regulated similarly to physiological bone mineralization. Mineralization also occurs in apoptotic cells and vesicles found in the intracellular and extracellular matrices (18). The latter is the most common source of breast MCs, suggesting that mammary MC formation is an active secretory rather than a passive process, such as in degeneration or necrosis $(19,20)$.

When MCs are associated with epithelial cells, it is possible that, under specific circumstances, a subpopulation of epithelial breast cells may undergo epithelial-to-mesenchymal transition (EMT) and then switch to osteoblast-like phenotypes (21). In concordance to this rationale, other reports demonstrated that the expression of certain osteogenic proteins, such as osteopontin and bone morphogenetic protein 2, is upregulated in breast cancer (22-27).

While these osteogenic features are well-documented in invasive breast cancer and in breast cancer cell lines in vitro, the reports on EMT features are scarce; changes related to mineralization and osteogenic model were reported in proliferative and precursor epithelial lesions associated with MCs (15). Although discrimination according to biochemical properties could improve the predictive value of MCs in mammograms, this requires biopsies providing sufficient samples for chemical determination, and this procedure is currently designed for diagnostic purposes only. An improvement in this setting would be using mass spectrometry profile from fine-needle aspiration samples $(28,29)$.

Unlike type I MCs, type II MCs may also be associated with malignant entities; therefore, the presence of casting-type HA MCs raises suspicion. The problem is that HA MCs are also frequently associated with benign entities $(30,31)$.

\section{Radiological classification of MCs}

MCs are commonly seen on mammograms and, therefore, there are well-described radiological patterns that help distinguish benign from potentially malignant calcifications (32). According to the fifth edition of the Breast Imaging Reporting and Data System (BI-RADS), MCs are classified as benign and suspicious. Benign calcifications on mammography are typically more extensive, coarser, rounder with smooth margins, and more easily seen than malignant calcifications (Fig. 2; Table II). Calcifications associated with malignancy are usually small in size and often require magnification to be well-visualized. MCs must be described according to morphology and distribution (33).
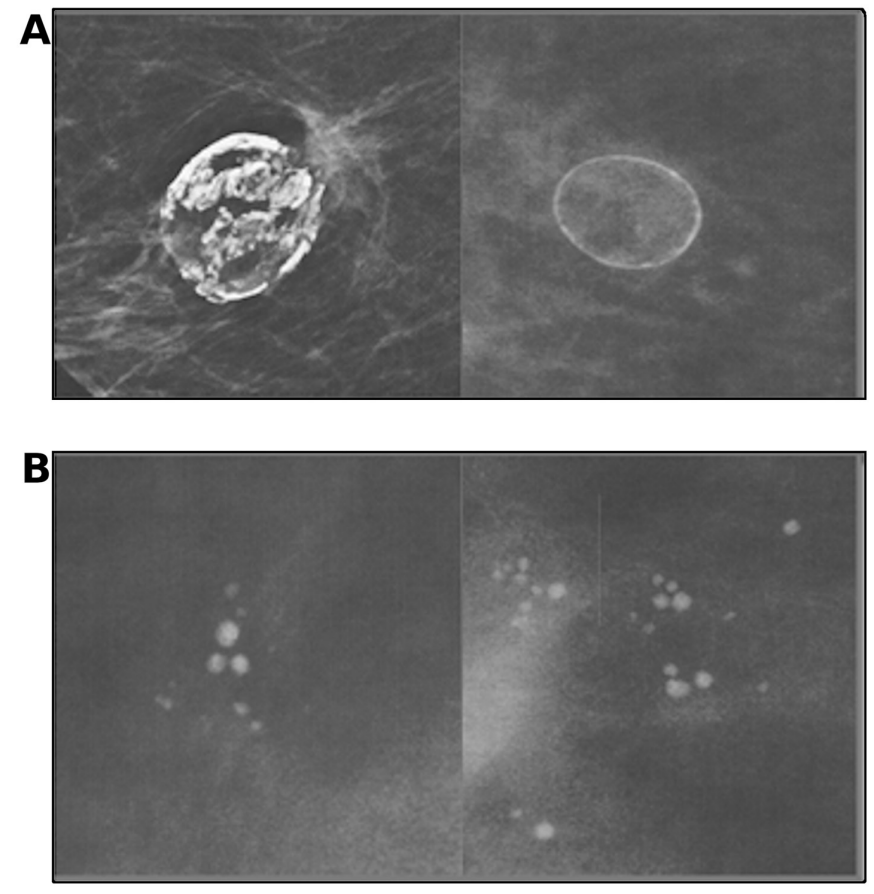

Figure 2. Examples of typically benign calcifications. Digital zoom mammographic projection images show (A) rim calcifications: Lucent-centered (left) and eggshell (right) calcifications have been combined into one category; and (B) round calcifications: Round (left) and punctate (right) calcifications have been combined into one category.

Suspicious morphology includes coarse heterogeneous appearance, amorphous nature, fine pleomorphic elements, and fine linear branching calcifications. The risk of malignancy of each descriptor are 13,27, 50 and $78 \%$, respectively (Fig. 3). The five distribution categories are diffuse, segmental, regional, grouped and linear (5).

Calcifications are extremely frequent alterations seen in $80 \%$ of mammograms, they mostly reflect a benign process and are not associated with cancer. However, when they present as small particles (MCs), grouped and polymorphic, they may be associated with malignancy (34-36). Mammography is the main modality used to evaluate these alterations. MRI has shown encouraging data in the diagnosis of breast cancer; however, its role in the evaluation of MCs remains under study, most likely because of low-grade lesions that exhibit little angiogenesis. Breast ultrasonography was also shown to lack the ability to detect MCs, as breast nodules are the lesions most often identified using ultrasonography (34-36). 

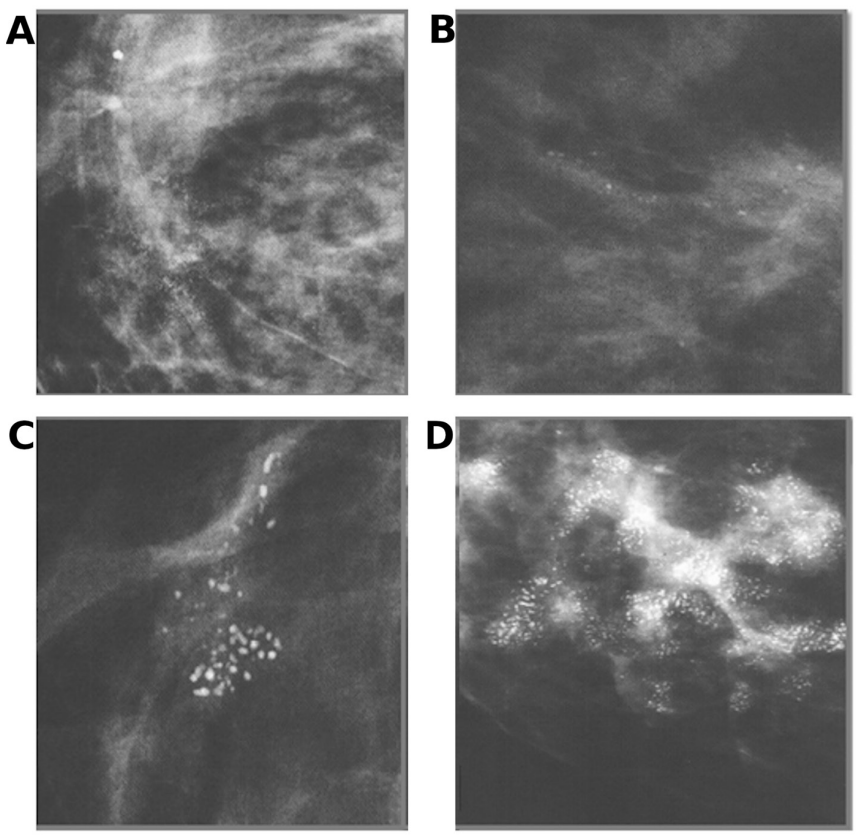

Figure 3. Calcifications with suspicious morphology. Digital zoom mammographic projection images show calcifications associated with increasing risk for malignancy: (A) Coarse heterogeneous, (B) amorphous, (C) fine pleomorphic and (D) fine linear or fine linear branching.

\section{MC management and biopsy with radiological guidance}

MCs represent a challenge in terms of detection and interpretation. Small, grouped MCs are easy to miss and difficult to interpret. When MCs do not exhibit benign features on screening mammography, the patient must be recalled for assessment using alternative mammogram views $(32,34)$. Final radiological evaluation leads to three possibilities: i) On magnified or supplementary views, MCs may be considered typically benign and classified as BI-RADS 2, and then follow-up is recommended; ii) MCs that are rounded and grouped may be classified as BI-RADS 3, and another imaging 6 months later for re-evaluation of stability is sufficient; and iii) when MCs exhibit suspicious morphological characteristics, they are classified as BI-RADS 4, and stereotactic biopsy is recommended (33).

Breast image-guided biopsies vary immensely technically. The size of the biopsy needle varies according to local protocols, device availability and indications on a case-by-case basis. Open excision is the final option, and the most frequent diagnostic procedure employed worldwide is a core biopsy. More recently, vacuum-assisted breast biopsy (VABB) has become more common $(32,37)$. Core biopsies are usually performed using a $14 \mathrm{G}$ needle, which may be helpful to establish a benign diagnosis, such as microcystic or fibroadenomatoid change, or a malignant diagnosis of invasive cancer; however, this method may underestimate the nature of the disease in $~ 27 \%$ of cases when ductal carcinoma in situ (DCIS) and indeterminate lesions, such as atypia, are present $(32,37)$.

VABB uses a large caliber device ranging from 7 to $12 \mathrm{G}$. In most cases, a $14 \mathrm{G}$ biopsy is sufficient to make the diagnosis; exceptions are made when there are very small $(<5 \mathrm{~mm})$ or scattered microcalcified clusters, and VABB is preferred in the first instance $(32,34)$. In cases of MCs $>10 \mathrm{~mm}$ in size, a core needle biopsy can be used alternatively $(35,36)$.

When MCs are detected only on mammography or in non-nodular lesions, they usually require stereotactic guidance to ensure a safe biopsy procedure, proper investigation of MCs, and provide high-quality samples (38). The number and width of the fragments are larger, comparable to samples obtained in surgical procedures (32), and MCs are easily retrieved according to Meyer et al (39). Standard automated needle devices were able to retrieve MCs within the sample in $90.8 \%$ of the cases compared with $95-100 \%$ in samples extracted using a vacuum-assisted device $(11,39)$. This procedure allows pathologists to gain access to more tissue for analysis, improving diagnostic accuracy. It is estimated that at least two cores with at least five calcium specks are sufficient (38).

The false-negative rate with VABB is low, and the correlation with final diagnosis is high, although there remains some degree of underestimation $(\sim 12 \%)$. Consequently, the likelihood of subsequent alteration in the grade of the lesion on follow-up is minimal. This procedure also has the advantage of achieving a definitive diagnosis with a single pass, reducing the duration of the procedure, as the needle is introduced only once and the samples are large in volume (38).

Other advantages are the allowance for placement of a titanium marker at the biopsy site and a lower diagnostic underestimation rate compared with core needle biopsy (39). Although vacuum-assisted large core biopsy provides a number of benefits, its cost is substantially greater compared with that of a $14 \mathrm{G}$ biopsy. Furthermore, this device is not yet available in many countries and underdeveloped regions. The surgical approach using segmental excision remains the only alternative in several facilities worldwide. In such cases, surgical and pathological management are crucial for determining the presence of MCs within the parenchyma $(32,41)$.

\section{Sending MC specimens to the pathology laboratory}

It is crucial to obtain X-ray images of all fragments obtained from breast biopsies for MC evaluation in order to select those with MCs; the presence, size and morphology of the MCs should match the mammography findings (Fig. 4A). Once the fragments containing MCs are identified on X-ray, it is usually recommended to keep the affected fragments in vials separate from the others. If possible, it is advisable to send the original $\mathrm{X}$-ray image taken from the fragments along with the samples to the pathology laboratory. This procedure is meant to help the pathologist identify MCs and determine which lesions are associated with them (38).

Pathological management and report of MC-related lesions. In this setting, the pathologist will receive two vials from the same breast lesion, one containing fragments with MCs and the other containing the remaining fragments (supposedly without MCs). These should be distinct paraffin-embedded blocks with separate slides. It is important to remember that, although the fragments are sent to the pathologist separately, they originate from the same region or lesions and should be assessed as a single lesion with a primary diagnosis. The findings are usually combined in one conclusion, as there may 


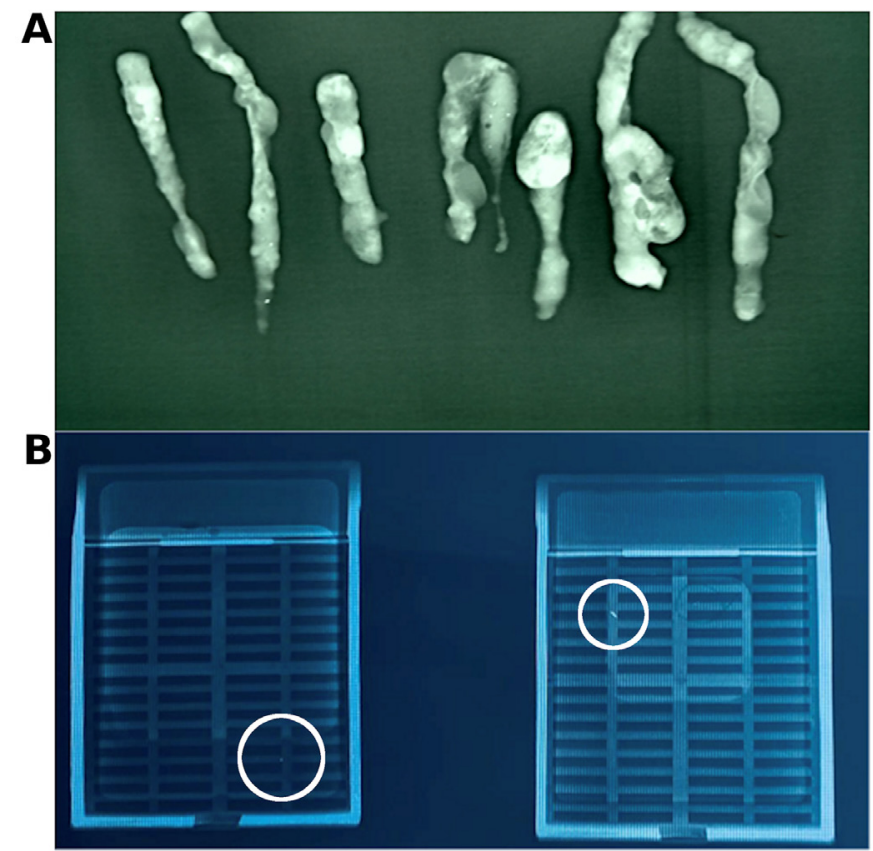

Figure 4. (A) X-ray image of fragments obtained in breast biopsies (B) Paraffin block X-ray may detect some of the MCs (white circles) and assure that the tissue sampled and embedded still contains the MCs. MCs, microcalcifications.

be some confusion when patients and, possibly, generalist physicians are confronted with the dichotomy of two distinct (and sometimes opposite) findings in the final report, since fragments without MCs often do not contain morphological indications of any malignant lesion.

It is crucial to identify MCs and report their presence in the pathology report, as it assures that the biopsied area was the actual target of the examination. When possible, it should be confirmed whether MCs are irregular or birefringent, delicate MCs. When the fragments labeled as 'with MCs' are microscopically analyzed, it may be the case that MCs are not easily identifiable, as they may lie deeper in the paraffin block, or they may be lost during sample processing, while certain technical measures mentioned below may resolve this problem (42).

Asking for subsequent sections of the block may be recommended, particularly if the lesion appears to be benign or if it must be ascertained that the area of the MCs was sampled appropriately. For suspicious lesions, caution must be taken when leveling the block, as some of the putative lesion may be lost, or later immunohistochemical evaluation may be compromised (43).

Large cysts with fluid secretion and benign entities commonly display irregular cavities, such as scar tissue and fat necrosis secretions associated with MCs, that disappear on histological examination. This may be due to the fact that they are lost during histological section preparation, because the MCs may not be rigidly attached to the tissues; therefore, after sectioning, they may no longer be identifiable on hematoxylin and eosin-stained slides. When even deeper levels fail to demonstrate the remaining MCs in this scenario, an X-ray of the blocks may be requested (Fig. 4B). The paraffin block $\mathrm{X}$-rays may reveal the MCs and ensure that the tissue sampled and embedded on the block in question is the targeted one, and if the paraffin block X-ray image does not reveal any MCs, the conclusion is that they were likely lost during processing. A caution note must be included in the report when this is the case, so that clinicians can double-check by performing a post-procedure mammogram to determine whether the targeted MCs were removed entirely $(38,39)$.

An alternative that may be very useful is to examine the hematoxylin and eosin-stained slides obtained under polarized light, which may reveal evidence of type I MCs. It is crucial to remember that type I MCs are often associated with benign lesions, and any suspicion of malignancy must be checked out by performing detailed assessment and correlation with radiological aspects of the MCs $(6,8)$

In addition, locating the MCs may not always be the most significant challenge; instead, the challenge may lie with defining lesions according to width. Pleomorphic calcifications may be the only remaining feature of a previous DCIS lesion. Occasionally, DCIS may undergo regression with fibrosis and elastosis, and it may be challenging to locate viable epithelial cells to achieve a definitive diagnosis $(6,8)$. Large fibrotic ducts with no apparent epithelial covering must be interpreted as suspicious if associated with significant central MCs. Subsequent levels of the block may reveal a suitable layer of representative ductal cells and facilitate the assessment $(6,8)$.

\section{MCs in breast cancer}

MCs are associated with premalignant and proliferative breast disease, and their identification facilitates the prevention of invasive disease. MCs may play a prognostic role in invasive carcinomas. Carcinomas with MCs appear to have worse outcomes compared with those without MCs (45). Furthermore, fine linear branching MCs have been associated with worse outcomes compared with non-linear MCs $(3,31,42,43)$.

In invasive carcinomas, the presence of MCs confers worse outcome in terms of parameters such as 8-year survival rate, tumor width and lymph node involvement (45). Since Tabár et al (46) examined the possible prognostic role of MCs in invasive breast cancer in 2000, several contributions have accumulated robust evidence to suggest that the presence of MCs, particularly those of the casting-type morphology in invasive breast cancer, is associated with poor outcome, higher risk of mortality, propensity for recurrence and HER-2-positive status. Among these studies are robust meta-analyses pooling odds ratios from more extensive series (47-49).

These concepts may shed light on the substantial importance of identifying MCs within an invasive tumor on pathological reports, even when doing so does not appear to be relevant information in the context of an already invasive nodular lesion. Since the presence, composition and type of MCs in breast lesions may influence the outcome, it is crucial that the presence and morphological characteristics of MCs are accurately reported to direct appropriate treatment decision-making. Some challenging prospective clinical studies in low-grade and intermediate-grade DCIS (such as LORIS, LORD and COMET) are testing the hypothesis of conservative treatment alternatives for such lesions $(11,47-49)$. 


\section{MCs in specific clinical scenarios}

MCs in high-density breast tissue. Diffusely dense breasts may be challenging to analyze on mammography, and some efforts have been made to improve the visualization of MCs on ultrasound imaging. However, the poor reproducibility of this method and limitations inherent to ultrasonic detection systems remain impediments to adequate implementation (50).

MCs in young patients. As most screening guidelines generally exclude women aged $<40$ years, few studies have verified the characteristics of breast MCs in young patients. This is more frequent in Asia. Fushimi et al (51) reviewed 403 mammograms from patients younger than 40 years and compared them to older patients (aged 40-74 years). Women aged $<40$ years were more likely to be recalled for MCs rather than for nodular lesions. The most frequent MC forms were small round and segmental MCs.

MCs in male breasts. In men, breast lesions are usually radiographically examined after the appearance of clinical symptoms and, therefore, MCs are not the first sign as in screening mammograms in women. Breast lesions in men were found to be associated with MC in $29 \%$ of cases reported by Mathew et al (52). However, they are not typically the main index of suspicion. In men, MCs are associated with benign as well as malignant lesions and they are less numerous, coarser and rounder compared with those in female breast tissue. Fat necrosis after trauma is a common benign cause associated with dystrophic MCs in men (52).

$M C s$ in the neoadjuvant setting. Invasive carcinomas with and without in situ components may contain MCs associated with the extension of the original lesion; however, these are not a reliable source of information regarding the amount of viable tumor tissue following neoadjuvant therapy. According to the tumor response to neoadjuvant treatment and the presence of necrosis and fibrosis, the number and extent of MCs may remain stable, decrease, or sometimes even increase $(52,53)$. Previous studies concluded that the pattern of MCs after neoadjuvant therapy on mammography and MRI examination tended to overestimate the true extent of the remaining pathological lesion $(52,53)$.

Most authors have found no significant association between complete pathological response and MC patterns following neoadjuvant therapy. There is also evidence of MCs being associated with benign changes in this setting (54). As a result, complete excision of all indeterminate or suspicious calcifications remains the standard practice (55).

\section{Conclusions}

The importance of MCs in cancer detection has become apparent over the last 20 years. With novel digital and technological detection methods being made available, the percentage of detected cases bearing MCs has increased. Currently, 30-50\% of non-palpable breast cancers are detected solely by MC identification on mammography (2-4). However, MCs represent a challenge regarding detection and interpretation. Therefore, radiological and pathological evaluations and expertise in pathoradiological correlations are crucial for accurately diagnosing these lesions. Contributions in this field are crucial to enhance the accuracy of the interpretation of radiological and pathological findings. The type and composition of MCs, including determination of their biochemical nature, could improve their predictive value. Novel potential markers of malignancy in breast lesions may be uncovered from the MC profile in the future.

\section{Acknowledgements}

Not applicable.

\section{Funding}

No funding was received.

\section{Availability of data and materials}

Not applicable.

\section{Authors' contributions}

AFL designed the study and wrote the manuscript. KCKP and CCBAN contributed to manuscript writing, images and tables. AFVF critically reviewed the manuscript for important intellectual content. MSDAC edited the information regarding the association of the context of the radiological reports. All the authors have read and approved the final version of the manuscript. AFL and AFVF confirm the authenticity of all the raw data of the paper.

\section{Ethics approval and consent to participate}

Not applicable.

\section{Patient consent for publication}

Not applicable.

\section{Competing interests}

The authors declare that they have no competing interests.

\section{References}

1. Salomon A: Beiträge zur Pathologie und Klinik der Mammokarzinome (In German). Arch Klin Chir 101: 573-668, 1913.

2. Leborgne R: Diagnosis of tumors of the breast by simple roentgenography; calcifications in carcinomas. Am J Roentgenol Radium Ther 65: 1-11, 1951.

3. Gülsün M, Demirkazik FB and Ariyürek M: Evaluation of breast microcalcifications according to Breast Imaging Reporting and Data System criteria and Le Gal's classification. Eur J Radiol 47: 227-231, 2003.

4. Venkatesan A, Chu P, Kerlikowske K, Sickles EA and Smith-bindman R: Positive predictive value of specific mammographic findings according to reader and patient va riables. Radiology 250: 648-657, 2009.

5. Rominger $\mathrm{M}$, Wisgickl $\mathrm{C}$ and Timmesfeld $\mathrm{N}$ : Breast microcalcifications as type descriptors to stratify risk of malignancy: A systematic review and meta-analysis of 10665 cases with special focus on round/punctate microcalcifications. Rofo 184: 1144-1152, 2012. 
6. Sharma T, Radosevich JA, Pachori G and Mandal CC: A Molecular View of Pathological Microcalcification in Breast Cancer. J Mammary Gland Biol Neoplasia 21: 25-40, 2016.

7. Henrot P, Leroux A, Barlier C and Génin P: Breast microcalcifications: The lesions in anatomical pathology. Diagn Interv Imaging 95: 141-152, 2014.

8. Frappart L, Boudeulle M, Boumendil J, Lin HC, Martinon I, Palayer C, Mallet-Guy Y, Raudrant D, Bremond A, Rochet Y, et al: Structure and composition of microcalcifications in benign and malignant lesions of the breast: Study by light microscopy, transmission and scanning electron microscopy, microprobe analysis, and X-ray diffraction. Hum Pathol 15: 880-889, 1984.

9. Haka AS, Shafer-Peltier KE, Fitzmaurice M, Crowe J, Dasari RR and Feld MS: Identifying microcalcifications in benign and malignant breast lesions by probing differences in their chemical composition using Raman spectroscopy. Cancer Res 62 : 5375-5380, 2002.

10. Trop I, David J, El Khoury M, Gautier N, Gaboury L and Lalonde L: Microcalcifications around a collagen-based breast biopsy marker: Complication of biopsy with a percutaneous marking system. AJR Am J Roentgenol 197: W353-357, 2011.

11. Liberman L, Smolkin JH, Dershaw DD, Morris EA, Abramson AF and Rosen PP: Calcification retrieval at stereotactic, 11-gauge, directional, vacuum-assisted breast biopsy. Radiology 208: 251-260, 1998

12. Castellaro AM, Tonda A, Cejas HH, Ferreyra H, Caputto BL, Pucci OA and Gil GA: Oxalate induces breast cancer. BMC Cancer 15: 761, 2015.

13. Radi MJ: Calcium oxalate crystals in breast biopsies. An overlooked form of microcalcification associated with benign breast disease. Arch Pathol Lab Med 113: 1367-1369, 1989.

14. Shih C, Padhy LC, Murray M and Weinberg RA: Transforming genes of carcinomas and neuroblastomas introduced into mouse fibroblasts. Nature 290: 261-264, 1981

15. Scimeca M, Giannini E, Antonacci C, Pistolese CA, Spagnoli LG and Bonanno E: Microcalcifications in breast cancer: An active phenomenon mediated by epithelial cells with mesenchymal characteristics. BMC Cancer 14: 286, 2014.

16. Morgan MP, Cooke MM and McCarthy GM: Microcalcifications associated with breast cancer: An epiphenomenon or biologically significant feature of selected tumors? J Mammary Gland Biol Neoplasia 10: 181-187, 2005.

17. Owen TA, Aronow M, Shalhoub V, Barone LM, Wilming L, Tassinari MS, Kennedy MB, Pockwinse S, Lian JB and Stein GS Progressive development of the rat osteoblast phenotype in vitro: Reciprocal relationships in expression of genes associated with osteoblast proliferation and differentiation during formation of the bone extracellular matrix. J Cell Physiol 143: 420-430, 1990

18. Huang W, Yang S, Shao J and Li YP: Signaling and transcriptional regulation in osteoblast commitment and differentiation. Front Biosci 12: 3068-3092, 2007.

19. Hassan MQ, Maeda Y, Taipaleenmaki H, Zhang W, Jafferji M, Gordon JA, Li Z, Croce CM, van Wijnen AJ, Stein JL, et al: miR-218 directs a Wnt signaling circuit to promote differentiation of osteoblasts and osteomimicry of metastatic cancer cells. J Biol Chem 287: 42084-42092, 2012.

20. O'Grady S and Morgan MP: Microcalcifications in breast cancer: From pathophysiology to diagnosis and prognosis. Biochim Biophys Acta Rev Cancer 1869: 310-320, 2018

21. Menck K, Scharf C, Bleckmann A, Dyck L, Rost U, Wenzel D, Dhople VM, Siam L, Pukrop T, Binder C, et al: Tumor-derived microvesicles mediate human breast cancer invasion through differentially glycosylated EMMPRIN. J Mol Cell Biol 7: 143-153, 2015.

22. Abate-Shen C: Deregulated homeobox gene expression in cancer: Cause or consequence? Nat Rev Cancer 2: 777-785, 2002.

23. Davies SR, Watkins G, Douglas-Jones A, Mansel RE and Jiang WG: Bone morphogenetic proteins 1 to 7 in human breast cancer, expression pattern and clinical/prognostic relevance. J Exp Ther Oncol 7: 327-338, 2008.

24. Jin H, Pi J, Huang X, Huang F, Shao W, Li S, Chen Y and Cai J: BMP2 promotes migration and invasion of breast cancer cells via cytoskeletal reorganization and adhesion decrease: An AFM investigation. Appl Microbiol Biotechnol 93: 1715-1723, 2012.

25. Liu F, Bloch N, Bhushan KR, De Grand AM, Tanaka E, Solazzo S Mertyna PM, Goldberg N, Frangioni JV and Lenkinski RE: Humoral bone morphogenetic protein 2 is sufficient for inducing breast cancer microcalcification. Mol Imaging 7: 175-186, 2008
26. Bellahcène A, Merville MP and Castronovo V: Expression of bone sialoprotein, a bone matrix protein, in human breast cancer. Cancer Res 54: 2823-2826, 1994.

27. Bellahcène A and Castronovo V: Increased expression of osteonectin and osteopontin, two bone matrix proteins, in human breast cancer. Am J Pathol 146: 95-100, 1995.

28. Barman I, Dingari NC, Saha A, McGee S, Galindo LH, Liu W, Plecha D, Klein N, Dasari RR and Fitzmaurice M: Application of Raman spectroscopy to identify microcalcifications and underlying breast lesions at stereotactic core needle biopsy. Cancer Res 73: 3206-3215, 2013.

29. Saha A, Barman I, Dingari NC, McGee S, Volynskaya Z, Galindo LH, Liu W, Plecha D, Klein N, Dasari RR, et al: Raman spectroscopy: A real-time tool for identifying microcalcifications during stereotactic breast core needle biopsies. Biomed Opt Express 2: 2792-2803, 2011.

30. Cox R: Cellular and molecular basis of mammary microcalcifications. PhD dissertation, Royal College of Surgeons in Ireland. https://doi.org/10.25419/rcsi.10804970.v1, 2011.

31. Cox RF and Morgan MP: Microcalcifications in breast cancer: Lessons from physiological mineralization. Bone 53: 437-450, 2013.

32. Wilkinson L, Thomas V and Sharma N: Microcalcification on mammography: Approaches to interpretation and biopsy. Br J Radiol 90: 20160594, 2017.

33. Sickles EA, D'Orsi CJ and Bassett LW: ACR BI-RADS Mammography. In: ACR BI-RADS Atlas, Breast Imaging Reporting and Data System. 5th Edition. American College of Radiology, Reston, VA, pp134-136, 2013.

34. Uematsu T, Yuen S, Kasami M and Uchida Y: Dynamic contrast-enhanced MR imaging in screening detected microcalcification lesions of the breast: Is there any value? Breast Cancer Res Treat 103: 269-281, 2007

35. Bazzocchi M,Zuiani C,Panizza P,Del Frate C, Soldano F, Isola M, Sardanelli F, Giuseppetti GM, Simonetti G, Lattanzio V, et al: Contrast-enhanced breast MRI in patients with suspicious microcalcifications on mammography: Results of a multicenter trial. AJR Am J Roentgenol 186: 1723-1732, 2006.

36. Cilotti A, Iacconi C, Marini C, Moretti M, Mazzotta D, Traino C, Naccarato AG, Piagneri V, Giaconi C, Bevilacqua G and Bartolozzi C: Contrast-enhanced MR imaging in patients with BI-RADS 3-5 microcalcifications. Radiol Med 112: 272-286, 2007.

37. Pfarl G, Helbich TH, Riedl CC, Wagner T, Gnant M, Rudas M and Liberman L: Stereotactic 11-gauge vacuum-assisted breast biopsy: A validation study. AJR Am J Roentgenol 179: 1503-1507, 2002.

38. Esen G, Tutar B, Uras C, Calay Z, İnce Ü and Tutar O Vacuum-assisted stereotactic breast biopsy in the diagnosis and management of suspicious microcalcifications. Diagn Interv Radiol 22: 326-333, 2016

39. Meyer JE, Smith DN, DiPiro PJ, Denison CM, Frenna TH, Harvey SC and Ko WD: Stereotactic breast biopsy of clustered microcalcifications with a directional, vacuum-assisted device. Radiology 204: 575-576, 1997.

40. Badan GM, Roveda Júnior D, Piato S, Fleury EF, Campos MS, Pecci CA, Ferreira FA and D'Ávila C: Diagnostic underestimation of atypical ductal hyperplasia and ductal carcinoma in situ at percutaneous core needle and vacuum-assisted biopsies of the breast in a Brazilian reference institution. Radiol Bras 49: 6-11, 2016.

41. Houssami N, Ciatto S, Ellis I and Ambrogetti D: Underestimation of malignancy of breast core-needle biopsy: Concepts and precise overall and category-specific estimates. Cancer 109: 487-495, 2007.

42. Tornos C, Silva E, el-Naggar A and Pritzker KP: Calcium oxalate crystals in breast biopsies. The missing microcalcifications. Am J Surg Pathol 14: 961-968, 1990.

43. D'Orsi CJ, Reale FR, Davis MA and Brown VJ: Is calcium oxalate an adequate explanation for nonvisualization of breast specimen calcifications? Radiology 182: 801-803, 1992.

44. Ling H, Liu ZB, Xu LH, Xu XL, Liu GY and Shao ZM: Malignant calcification is an important unfavorable prognostic factor in primary invasive breast cancer. Asia Pac J Clin Oncol 9: 139-145, 2013.

45. Bonfiglio R, Scimeca M, Urbano N, Bonanno E and Schillaci O: Breast microcalcifications: Biological and diagnostic perspectives. Future Oncol 14: 3097-3099, 2018

46. Tabár L, Chen HH, Duffy SW, Yen MF, Chiang CF, Dean PB and Smith RA: A novel method for prediction of long-term outcome of women with T1a, T1b, and 10-14 mm invasive breast cancers: A prospective study. Lancet 355: 429-433, 2000. 
47. Elias SG, Adams A, Wisner DJ, Esserman LJ, van't Veer LJ, Mali WP, Gilhuijs KG and Hylton NM: Imaging features of HER2 overexpression in breast cancer: A systematic review and meta-analysis. Cancer Epidemiol Biomarkers Prev 23: 1464-1483, 2014

48. Nyante SJ, Lee SS, Benefield TS, Hoots TN and Henderson LM: The association between mammographic calcifications and breast cancer prognostic factors in a population-based registry cohort. Cancer 123: 219-227, 2017.

49. Zheng K, Tan JX, Li F, Wei YX, Yin XD, Su XL, Li HY, Liu QL, Ma BL, Ou JH, et al: Relationship between mammographic calcifications and the clinicopathologic characteristics of breast cancer in Western China: A retrospective multi-center study of 7317 female patients. Breast Cancer Res Treat 166: 569-582, 2017.

50. Ouyang YL, Zhou ZH, Wu WW, Tian J, Xu F, Wu SC and Tsui PH: A review of ultrasound detection methods for breast microcalcification. Math Biosci Eng 16: 1761-1785, 2019.

51. Fushimi A, Fukushima N, Suzuki T, Kudo R and Takeyama H: Features of microcalcifications on screening mammography in young women. Asian Pac J Cancer Prev 19: 3591-3596, 2018.
52. Mathew J, Perkins GH, Stephens T, Middleton LP and Yang WT: Primary breast cancer in men: Clinical, imaging, and pathologic findings in 57 patients. AJR Am J Roentgenol 191: 1631-1639, 2008.

53. Weiss A, Lee KC, Romero Y, Ward E, Kim Y, Ojeda-Fournier H, Einck J and Blair SL: Calcifications on mammogram do not correlate with tumor size after neoadjuvant chemotherapy. Ann Surg Oncol 21: 3310-3316, 2014

54. Adrada BE, Huo L, Lane DL, Arribas EM, Resetkova E and Yang W: Histopathologic correlation of residual mammographic microcalcifications after neoadjuvant chemotherapy for locally advanced breast cancer. Ann Surg Oncol 22: 1111-1117, 2015.

55. Feliciano Y, Mamtani A, Morrow M, Stempel MM, Patil S and Jochelson MS: Do Calcifications Seen on Mammography After Neoadjuvant Chemotherapy for Breast Cancer Always Need to Be Excised? Ann Surg Oncol 24: 1492-1498, 2017. International (CC BY-NC-ND 4.0) License. 